Roman Kozhan 


\title{
Non-Additive Anonymous Games
}

\author{
Roman Kozhan* \\ Warwick Business School
}

\begin{abstract}
This paper introduces a class of non-additive anonymous games where agents are assumed to be uncertain (in the sense of Knight) about opponents' strategies and about the initial distribution over players' characteristics in the game. These uncertainties are modelled by non-additive measures or capacities. The CournotNash equilibrium existence theorem is proven for this class of games. It is shown that the equilibrium distribution can be symmetrized under milder conditions than in the case of additive games. In particular, it is not required for the space characteristics to be atomless under capacities. The set-valued map of the Cournot-Nash equilibria is upper-semicontinuous as a function of initial beliefs of the players for non-additive anonymous games.
\end{abstract}

Key words: anonymous game, uncertainty, non-additive measure, capacity.

JEL Classification: C72, D81

\section{Introduction}

Standard non-cooperative game theory assumes that players involved in a game behave strategically and rationally. They form beliefs about their opponents' actions and choose their best response given those beliefs. In most complex situations it is difficult (or even impossible) to guess the action of an opponent precisely, therefore beliefs are usually modelled in a probabilistic manner. That is, a player would construct

*Address for correspondence: Warwick Business School, University of Warwick, Coventry, CV4 7AL, UK; e-mail: Roman.Kozhan@wbs.ac.uk 
a probability distribution which represents her beliefs about future actions of other players.

This assumption however is not realistic and too restrictive in many circumstances. There is a growing body of literature which demonstrates that individuals do not form probabilistic beliefs in a wide range of real-world situations. A classical example is the Ellsberg's Paradox (Ellsberg 1961) which shows that individual's preferences cannot be supported by a probability measure as suggested by the subjective expected utility model (Savage 1954).

The main reason for this is the lack of information about future outcomes. The psychological nature of perceiving uncertainty generated by the incompleteness of information turns out to be much more richer than it could be explained by a probability distribution. The notion of uncertainty was introduced back in 1921 by Frank Knight (Knight 1921) who proposed the distinction between those situations where individual's preferences can be described by a probabilistic model (risk) and where such models do not exist (uncertainty).

Uncertainty naturally would seem to increase as information becomes more complex. Generally in a game with one opponent a player will face less uncertainty than in the game with ten participants. As the number of players grows, it becomes crucial to be able to model uncertainty correctly. In this paper we focus on games with a large number of players who play under informational uncertainty. In such games players do not consider their opponents individually but rather look at their future actions in aggregate. Mas-Colell (1986) proposed an approach where players' beliefs about the aggregate structure of participants is modelled by a probability distribution and this distribution is a common knowledge. This assumption is very often unrealistic as in such big games players do not have precise information about the other participants. It is unlikely that all players in such a situation would be able to correctly estimate the probability and agree on this distribution. Can we precisely identify the distribution of the beliefs of all traders in London Stock Exchange? If individuals do not behave in accordance with the standard theory in simple situations, as in Elsberg's paradox, we cannot expect them to do so in more complex situations. This is the place where many different psychological biases enter the decision process which lead to paradoxical outcomes observed in real life games and experiments.

What is the alternative model for players' beliefs if we are not going to use a probability measure? A number of authors have introduced models which describe individuals' beliefs about future outcomes in the face of uncertainty by a non-additive 
measure (also called capacity or fuzzy measure). ${ }^{1}$ Within these frameworks a representative agent maximizes his preference functional, defined as a Choquet integral over a utility function of future payoffs.

Many empirical investigations provide strong evidence that individuals are not uncertainty neutral in real life and react to uncertainty differently than they do to risk. In the majority of cases people are uncertainty-averse (Ellsberg (1961), Camerer and Ho (1994), Tversky and Fox (1995)); however in some situations they tend to be uncertainty-loving as well (Curley and Yates (1989), Hogarth and Einhorn (1990)). The strength of the Choquet expected utility approach is that it makes it possible to model these different psychological attitudes to uncertainty. In fact, players can be optimistic, pessimistic or they can have other forms of mixed preferences. The notion of non-additive beliefs allows us to model simultaneously the imprecision of information and the attitude of participants to this imprecision. This brings substantial flexibility into modelling the structure of players' beliefs.

The notion of Knightian Uncertainty is not new in Game Theory. Dow and Werlang (1994), Klibanoff (1996), Lo (1996), Groes, Jacobsen, Sloth and Tranaes (1998), Eichberger and Kelsey (2000), Mukerji (1997), Marinacci (2000) and Ghirardato and LeBreton (2000) apply this approach to non-corporative games with a finite number of players and generalize the notion of Nash equilibria to games with uncertainty averse players. Again, players do not form beliefs about their opponents' strategies as a probability measure but their beliefs appear instead to be non-additive functions of subsets of pure strategies. In some special cases they can be interpreted as a set of possible mixed strategies that the opponent might play (see Klibanoff (1996), Lo (1996)). Recently Kozhan and Zarichnyi (2008) generalized the notion of Nash equilibria in capacities. They extended the results of Glycopantis and Muir (1999), Glycopantis and Muir (2000) and Aliprantis, Glycopantis and Puzzello (2006) regarding the continuity of expected payoffs and approximations of mixed strategies to the case of games under Knightian Uncertainty. Glycopantis and Muir (2008) provided an example of such games for the case of 2 players.

In this paper we develop a concept of Cournot-Nash (CN) equilibrium of anonymous games with players facing uncertainty. In its classical form an anonymous game is a generalization of a game with continuum players, introduced initially by Schmeidler (1973). Hart, Hildenbrand and Kohlberg (1974) and Mas-Colell (1986) developed the

\footnotetext{
${ }^{1}$ The reader is referred to Schmeidler (1989) and Gilboa and Schmeidler (1989) for a more detailed description of these models.
} 
concept of equilibrium based on the probability distribution of players' characteristics rather than on the set of players itself. The contribution of the present paper is to provide an extension of Mas-Colell's equilibrium to cases when the players' are not able to use a probabilistic distribution due to presence of uncertainty. Instead, they form their beliefs about the the characteristics of opponents in the form of non-additive measures or capacities. As a consequence, a whole range of new equilibria arises in this setup.

The extension of Nash's existence theorem for non-additive anonymous games is not as straightforward as in the case of games with finite number of players. The reason for this is that a Nash equilibrium of an anonymous game is a function of initial beliefs about agents' characteristics. In Mas-Colell (1986), this function is defined only on the space of all additive probability measures while its extension onto the space of capacities is required for non-additive anonymous games. This extension is provided below.

Introducing uncertainty into anonymous games allows to relax an assumption under which a $\mathrm{CN}$ equilibrium can be symmetrized. It is also shown that a non-additive equilibrium distribution can be symmetrized in case of atomic spaces and compact action spaces.

Another issue investigated in the paper is how the set of equilibria changes as the beliefs about the distribution over agents' characteristics change. Since the set of CN equilibria in anonymous games is a function of the initially defined distribution, some small distortions in beliefs may cause big deviations in equilibria. Thus, the question of the sensitivity of the set of CN equilibria to modeling assumptions is also addressed. Similar problems of the sensitivity of Nash equilibria in the case of additive games have been considered by Green (1984), Milgrom and Weber (1985), Kajii and Morris (1998) and others. In line with these papers we investigate the continuity of the equilibrum set in the topology of weak-* convergence on the space of non-additive measures.

The paper is organized as follows. In the next section definitions and preliminaries are provided. Section 2 provides some preliminary notions and definitions needed for the analysis and Section 3 gives the definition of a non-additive anonymous game and a motivating application. The existence theorem is stated and proven in Section 4. The symmetrization theorem is given in Section 5 and Section 6 provides the result about upper-semicontinuity of the equilibrium correspondence. Some concluding remarks are given in Section 7. 


\section{Preliminaries}

\section{Functor of capacities. $^{2}$}

Let $X$ be a compact Hausdorff space and $\mathcal{B}$ a $\sigma$-algebra of its Borel subsets. Denote by $C(X)$ the space of all continuous functions on $X$ endowed with the sup-norm topology.

Definition 2.1. A real-valued set function $\mu$ on $(X, \mathcal{B})$ is called a capacity if $\mu(\emptyset)=0$, $\mu(X)=1$ and $\mu(A) \leq \mu(B)$ for all $A \subseteq B, A, B \in \mathcal{B}$.

Definition 2.2. A capacity $\mu$ is upper-continuous if $\lim _{n \rightarrow \infty} \mu\left(A_{n}\right)=\mu\left(\bigcap_{n=1}^{\infty} A_{n}\right)$ for any non-increasing sequence of sets $\left\{A_{n}\right\} \subset \mathcal{B}$.

Capacities are often called non-additive measures as they differ from measures only in the additivity axiom.

For any capacity $\tau$ defined on a product of spaces $\left(X \times Y, \mathcal{B}_{X} \times \mathcal{B}_{Y}\right)$ we can define its marginals $\tau_{X}$ and $\tau_{Y}$ by $\tau_{X}(F)=\tau(F \times Y)$ and $\tau_{Y}(G)=\tau(X \times G)$ for $F \in \mathcal{B}_{X}$ and $G \in \mathcal{B}_{Y}$

We denote the set of all upper-continuous capacities on $X$ by $M(X)$, and according to Zhou's representation theorem (see Zhou (1998)) we can identify it with the set of all comonotonically additive, monotonic and continuous functionals on $C(X)$ by the formula

$$
\mu(f)=\int_{0}^{\infty} \mu(f \geq t) d t+\int_{-\infty}^{0}(\mu(f \geq t)-1) d t .
$$

The above integral is called the Choquet integral. ${ }^{3}$

The set $M(X)$ endowed with the weak-* topology is a compact Hausdorff space. The base of this topology consists of the set of form

$$
O\left(\mu_{0}, f_{1}, \ldots, f_{n}, \varepsilon\right)=\left\{\mu \in M(X):\left|\mu_{0}\left(f_{i}\right)-\mu\left(f_{i}\right)\right|<\varepsilon, i=1, \ldots, n\right\}
$$

where $\mu_{0} \in M(X), f_{1}, \ldots, f_{n} \in C(X)$ and $\varepsilon>0 .{ }^{4}$

\footnotetext{
${ }^{2}$ It is assumed that readers have some basic background in the theory of normal functors and categorical topology. For more information about topological functors see Eilenberg and Moore (1965), Zarichnyi (1990), Teleiko and Zarichnyi (1999)

${ }^{3}$ In the name of Gustave Choquet who introduced the notion of integral with respect to nonadditive measures. See Choquet (1953) for more detailed description of its properties. The theory of the Choquet integral is also described in Denneberg (1994).

${ }^{4}$ For spaces of probability measures weak and weak-* topologies coincide because of the reflexivity
} 
The map $M:$ Comp $\rightarrow$ Comp forms a covariant functor in the category Comp of compact Hausdorff spaces and their continuous maps. It maps a compact Hausdorff space $X$ to the spaces of all upper-continuous capacities $M(X)$. Its morphism (action on continuous functions between compact Hausdorff spaces) $M f: M(X) \rightarrow M(Y)$ is defined as follows: for given $f: X \rightarrow Y, M(f(\nu))(B)=\nu\left(f^{-1}(B)\right)$ for $\nu \in M(X), B$ a Borel set of $Y$. It is shown in Nykyforchyn and Zarichnyi (2006), this functor is weakly normal.

Continuous correspondences. Let $X$ and $Y$ be compact Hausdorff spaces. Hereafter we denote by $F: X \rightarrow Y$ a set-valued map or correspondence. If all values of $F$ are closed subsets of $Y$ we will use an alternative notation $F: X \rightarrow \exp (Y)$, where $\exp (Y)$ denotes the space of all closed subsets of $Y$ endowed with the Vietoris topology. A base of this topology consists of the sets of the form

$$
\left\langle U_{1}, \ldots, U_{n}\right\rangle=\left\{A \in \exp (Y): A \subset U_{1} \cup \ldots \cup U_{n} \text { and } A \cap U_{i} \neq \emptyset \text { for every } i\right\},
$$

where $U_{1}, \ldots, U_{n}$ run through the topology of $Y$ (see Michael (1951) for more detailed information).

Definition 2.3. A correspondence $F: X \rightarrow Y$ is upper-semicontinuous at $x_{0}$ if for each sequence $\left\{x_{n}\right\}_{n \in \mathbb{N}} \subset X$ convergent to $x_{0} \in X$ and a sequence $\left\{y_{n}\right\}_{n \in \mathbb{N}} \subset Y$ such that $y_{n} \in F\left(x_{n}\right)$ the condition $\lim _{n \rightarrow \infty} y_{n}=y_{0}$ implies $y_{0} \in F\left(x_{0}\right)$.

$F$ is upper-semicontinuous if it is upper-semicontinuous at all points $x \in X$.

Definition 2.4. A correspondence $F: X \rightarrow Y$ is lower-semicontinuous at $x_{0}$ if for each convergent to $x_{0} \in X$ sequence $\left\{x_{n}\right\}_{n \in \mathbb{N}} \subset X$ and a point $y_{0} \in F\left(x_{0}\right)$ there exists a sequence $\left\{y_{n}\right\}_{n \in \mathbb{N}}$ such that $y_{n} \in F\left(x_{n}\right)$ for each $n \in \mathbb{N}$ and $\lim _{n \rightarrow \infty} y_{n}=y_{0}$.

$F$ is lower-semicontinuous if it is lower-semicontinuous at all points $x \in X$.

Definition 2.5. A correspondence $F: X \rightarrow Y$ is continuous if it is upper- and lowersemicontinuous.

The following two lemmata state continuity properties of the intersection and the union correspondences.

Lemma 2.6. Let $X$ be a compact Hausdorff space. Then the map $\cap$ : $\exp (X) \times$ $\exp (X) \rightarrow \exp (X)$ is upper-semicontinuous.

of the space of continuous functions on a compact Hausdorff space. In the case of capacities it is not the case any more. We specify the topology on $M(X)$ in the way that its restriction on the space of probability measures $P(X) \subset M(X)$ coincides with the weak-* topology on $P(X)$. 
Proof. Let $\left\{A_{n}\right\}_{n \in \mathbb{N}}$ and $\left\{B_{n}\right\}_{n \in \mathbb{N}}$ be a sequences of closed subsets of $X$ such that $A_{n} \cap B_{n} \neq \emptyset$. Let $A=\lim _{n \rightarrow \infty} A_{n}$ and $B=\lim _{n \rightarrow \infty} B_{n}$. Consider the sequence $\left\{c_{n}\right\}_{n \in \mathbb{N}}$ of points from $A_{n} \cap B_{n}$ converges to the point $c_{0}$ and assume that $c \notin A \cap B$. Without loss of generality we can suppose that $c \notin A$. Since the space $X$ is compact and Hausdorff it is regular and therefore there exist two open sets $U$ and $V$ such that $c_{0} \in U, A \subset V$ and $U \cap V=\emptyset$. Starting from some number $n_{0}$ all points $c_{n}$ belong to the neighborhood $U$ of $c_{0}$ and, thus, do not belong to $V$. On the other hand, there exists $n_{1}$ such that for all $n>\max \left\{n_{0}, n_{1}\right\}$ all sets $A_{n}$ should be in the neighborhood $\langle V\rangle$ of the set $A$. It is, however, not true because at least one point $c_{n}$ from $A_{n}$ does not belong to $V$ and therefore $A_{n} \nsubseteq\langle V\rangle$. An alternative proof of this lemma can be also found in Teleiko and Zarichnyi (1999).

Lemma 2.7. Let $X$ be a compact Hausdorff space. Then the map $\cup$ : $\exp (X) \times$ $\exp (X) \rightarrow \exp (X)$ is continuous.

See Teleiko and Zarichnyi (1999) for the proof.

Let us recall the notion of support of capacities which is defined from the categorical topology point of view. Let $F$ be a covariant functor and $a \in F(X)$ for some compact Hausdorff space $X$.

Definition 2.8. The support of an element $a \in F X$ is the set

$$
\operatorname{supp}_{F}(a)=\cap\{A \subset X: A \text { is closed and } a \in F(A)\} \text {. }
$$

Intuitively, Definition 2.8 refers to the smallest closed subset of $X$ which carry all the capacity for subsets of $X$.

The reader is referred to Teleiko and Zarichnyi (1999) for the proof of the following lemma.

Lemma 2.9. Let $F$ be a monomorphic functor in the category Comp that preserves the intersections. Then the map $\operatorname{supp}_{F, X}: F(X) \rightarrow \exp (X)$ is lower-semicontinuous.

\section{Non-Additive Anonymous Games}

We extend the definition of anonymous games given in Mas-Colell (1986) and present the concept of a non-additive anonymous game.

Let $A$ be a non-empty compact Hausdorff space of actions. Every player is characterized by its continuous utility function $u: A \times M(A) \rightarrow \mathbb{R}$. Given an action $a \in A$ 
taken by a player with a utility function $u$ and the action distribution of all players $\nu \in M(A)$, the value $u(a, \nu)$ reflects the utility of the player for choosing the action a. Let us denote by $\mathcal{U}$ a compact set of characteristics of the players, i.e. a set of continuous utility functions on $A \times M(A)$ endowed with the supremum norm. A game with uncertain players is represented by a capacity $\mu$ on $\mathcal{U}$ which represents common beliefs about a true distribution over players' characteristic. The capacity reflects players' attitudes to uncertainty which is assumed to be present in the game. As it was mentioned above, a capacity in general is a non-additive measure and therefore we naturally call such games non-additive anonymous games. Let us also note that a probability measure is a special case of capacities which implies that the notion of Mas-Colell's anonymous game is a special case of the concept of non-additive games introduced here. The non-additive equilibrium introduced below generalizes the concept of equilibrium proposed by Hart et al. (1974) and Mas-Colell (1986) for additive anonymous games.

Definition 3.1. A capacity $\tau \in M(\mathcal{U} \times A)$ is called a Cournot-Nash $(C N)$ equilibrium capacity if:

i) $\tau_{\mathcal{U}}=\mu$;

ii) $\tau\left(\left\{(u, a): u\left(a, \tau_{A}\right) \geq u\left(a^{\prime}, \tau_{A}\right)\right.\right.$ for any $\left.\left.a^{\prime} \in A\right\}\right)=1$,

The notion of Mas-Colell's anonymous game is a generalized version of a Schmeidler's games with a continuum of players. Anonymity in the game arises since only the distribution of players' characteristics $\mu$ matters.

According to Mas-Colell's interpretation the distribution $\mu$ is an objective distribution of players' characteristics, known to all participants of the game. It can be understood as a mass assigned to every utility of the players involved in the game.

In this paper we assume, however, that the measure $\mu$ is non-additive and represents all players common beliefs about this distribution. As an example of such a situation let us consider a foreign exchange market consisting of customers who create demand and supply for domestic and foreign currencies and dealers who set prices and are essentially market-makers. The strategy for the dealers is to set bid and ask quotes for currencies to maximize their profits. On the one hand, the bid-ask spread should be big enough to be able to make a profit, but on the other hand the bigger it is the less customers will be willing to trade with the dealer. At the same time some customers may be informed about future changes in fundamentals or other macro variables. As stated in Lyons (2001): 
"Dealers know that when they trade with someone who is better informed, they can expect to lose money on the trade. If one could identify better-informed traders before trading, then this would not be a problem - dealers could choose not to trade, or could adjust price appropriately - but dealers typically cannot identify those who are better informed."

Thus, it is critical for dealers to predict the ratio of informed (inf) versus uninformed (unf) traders in the market. Let us assume that a dealer estimates the proportion of better-informed traders by $0<\pi<1$ but he is uncertain about this value. If he underestimates the true value and sets prices with a small bid-ask spread, he may lose money. In order to insure himself against underestimation of this proportion the dealer can make his decision by assuming that the fraction of informed traders is $\pi_{1}>\pi$. This will lead to an increase in the bid-ask spread which could decrease the number of customers willing to trade with him substantially. Thus, both cases are undesirable. If we assume that the dealer is uncertainty averse and his preferences satisfy the axioms given by Gilboa and Schmeidler (1989) then the dealer will behave in this situation according to the worst-case scenario. The capacity which describes the dealer's beliefs about the distribution of market participants is given by $\min \left\{p \delta_{\text {inf }}+(1-p) \delta_{\text {unf }}: p \in(\pi-\varepsilon, \pi+\varepsilon)\right\}$ for some positive (perhaps small) $\varepsilon$ which is subjectively determined. Hence, the dealer's belief about the agents' distributions is formed by a non-additive measure.

Another source of uncertainty which appears in this game is that concerning the opponents' actions. Again, in Mas-Colell's anonymous games beliefs about opponents' actions are modelled by a probability distributions which are reflected in equilibrium by the marginal $\tau_{A}$. In the game under uncertainty this marginal becomes a non-additive measure in general.

\section{$3.1 \quad$ Example}

Let us a consider simpler but more formal example. ${ }^{5}$ There is a continuum of players who will be randomly paired and will play a game where two opponents simultaneously choose one of two actions $L$ and $R$. Suppose there are two possible types of people in the population, the first like to match what their partner does and the second like to mismatch. Specifically, suppose the payoff matrix for this game is given as follows:

\footnotetext{
${ }^{5}$ I was advised with the example what I am very grateful for.
} 


\begin{tabular}{cc|c|c} 
& \multicolumn{3}{c}{ Player 2 } \\
& & $L$ & $R$ \\
\cline { 2 - 4 } Player 1 & $L$ & 1,0 & 0,1 \\
\cline { 2 - 4 } & $R$ & 0,1 & 1,0 \\
\cline { 2 - 4 } & & & \\
\hline
\end{tabular}

Each player cannot identify the type of his opponent. His 'expected' payoff from choosing to play either $L$ or $R$ depends on his beliefs about how other players in the population will play. In particular, if his beliefs about the distribution of action choices in the population is represented by the capacity $\tau_{A}$ over $\{L, R\}$ then we have the payoff for a player of type 1 , from playing $L$ is given by the Choquet integral

$$
u_{1}\left(L, \tau_{A}\right)=\tau_{A}(\{L\}) u_{1}(L, L)+\left(1-\tau_{A}(\{L\})\right) u_{1}(L, R)=\tau_{A}(\{L\}) .
$$

The expected payoff of a player of type 1 from playing $R$ is given by the Choquet integral

$$
u_{1}\left(R, \tau_{A}\right)=\tau_{A}(\{R\}) u_{1}(R, R)+\left(1-\tau_{A}(\{R\})\right) u_{1}(R, L)=\tau_{A}(\{R\}) .
$$

Similarly, the expected payoff of a player of type 2 from playing $L$ or $R$ are given by the respective Choquet integrals

$$
\begin{aligned}
& u_{2}\left(L, \tau_{A}\right)=\tau_{A}(\{R\}) u_{2}(L, R)+\left(1-\tau_{A}(\{R\})\right) u_{2}(L, L)=\tau_{A}(\{R\}) \\
& u_{2}\left(R, \tau_{A}\right)=\tau_{A}(\{L\}) u_{2}(R, L)+\left(1-\tau_{A}(\{L\})\right) u_{1}(R, R)=\tau_{A}(\{L\}) .
\end{aligned}
$$

A game is characterized by a capacity $\mu$ on $\mathcal{U}=\{1,2\}$. Let us assume that $\mu(\{1\})>0$ and $\mu(\{2\})>0$, that is, there are positive common beliefs (possibly non-additive) about the presence in the population of players of both types.

Let us consider some special cases of CN equilibria. Let

$$
\begin{aligned}
& \tau^{*}(\emptyset)=0 \text { and } \tau^{*}(\mathcal{U} \times A)=1 \\
& \tau^{*}(\{i, a\})=0 \text { for all }(i, a) \in \mathcal{U} \times A \\
& \tau^{*}(\{(1, L),(2, R)\})=1 \\
& \tau^{*}(\{(1, R),(2, L)\})=0 \\
& \tau^{*}(\{(1, L),(1, R)\})=\mu(\{1\}) \\
& \tau^{*}(\{(2, L),(2, R)\})=\mu(\{2\}) \\
& \tau^{*}(\{(1, L),(2, L)\})=1 \\
& \tau^{*}(\{(1, R),(2, R)\})=1 \\
& \tau^{*}((\mathcal{U} \times A) \backslash\{(i, a)\})=1 \text { for all }(i, a) \in \mathcal{U} \times A
\end{aligned}
$$


It is easy to see that $\tau^{*}$ is a capacity. Let us show that $\tau^{*}$ is also a CN equilibrium. First note that $\tau_{\mathcal{U}}^{*}(\{1\})=\tau^{*}(\{(1, L),(1, R)\})=\mu(\{1\})$ and $\tau_{\mathcal{U}}^{*}(\{2\})=\tau^{*}(\{(2, L),(2, R)\})=$ $\mu(\{2\})$. Finally, we have

$$
\begin{aligned}
& u_{1}\left(L, \tau_{A}^{*}\right)=\tau_{A}^{*}(\{L\})=\tau_{A}^{*}(\{R\})=u_{1}\left(R, \tau_{A}^{*}\right)=1 \\
& u_{2}\left(R, \tau_{A}^{*}\right)=\tau_{A}^{*}(\{L\})=\tau_{A}^{*}(\{R\})=u_{2}\left(L, \tau_{A}^{*}\right)=1 .
\end{aligned}
$$

Hence

$$
\tau^{*}\left(\left\{(u, a): u\left(a, \tau_{A}^{*}\right) \geq u\left(A, \tau_{A}^{*}\right)\right\}\right)=1
$$

as required.

Let us note that by construction $\tau_{A}(\{L\})=\tau_{A}(\{R\})=1$. This means that there exists an equilibrium where players are extremely optimistic and believe with probability (non-additive) one that the nature will always be on their side. Therefore, according to their beliefs, whatever they choose to play their payoff will be positive. This fact may seem counter-intuitive. However, human behavior in real life is also inconsistent with the classical notion of rationality. People buy lotteries knowing that on average (under additive probability) they will loose. This fact however does not prevent them from expecting to get a valuable prize. They are optimistic and this optimism makes their decisions not fully rational in the classical sense. This example illustrates how game theory can explain why people often do not behave in accordance with a ("additive") CN equilibrium.

In the same way we can derive a pessimistic equilibrium where players will always expect a zero payoff.

\section{CN Equilibria and the Existence Theorem}

In this section the first result we give is the existence theorem for CN equilibria in nonadditive anonymous games. To establish this we need the following auxiliary lemmata. Let

$$
\begin{gathered}
K\left(\tau_{A}\right)=\left\{(u, a): u\left(a, \tau_{A}\right) \geq u\left(A, \tau_{A}\right)\right\}, \\
B_{1}^{\prime}(\mu)=\left\{\tau^{\prime}: \tau_{\mathcal{U}}^{\prime}=\mu\right\}, \\
B_{1}^{\prime \prime}\left(\tau_{A}\right)=\left\{\tau^{\prime}: \tau_{A}^{\prime}=\tau_{A}\right\}, \\
B\left(\tau_{A}\right)=\left\{\tau^{\prime}: \tau^{\prime}\left(K\left(\tau_{A}\right)\right)=1\right\} .
\end{gathered}
$$

Lemma 4.1. The correspondence $K: M(A) \rightarrow \mathcal{U} \times A$ is upper-semicontinuous. 
Proof. Let us consider an arbitrary sequence $\left\{\nu_{n}\right\}_{n \in \mathbb{N}} \subset M(A)$ which converges to a capacity $\nu_{0} \in M(A)$ as $n \rightarrow \infty$ and assume that there exists a sequence $\left\{\left(u_{n}, a_{n}\right)\right\}_{n \in \mathbb{N}} \subset$ $\mathcal{U} \times A$ such that $\left(u_{n}, a_{n}\right) \rightarrow\left(u_{0}, a_{0}\right)$ with $\left(u_{n}, a_{n}\right) \in K\left(\nu_{n}\right)$ for every $n \in \mathbb{N}$ and $\left(u_{0}, a_{0}\right) \notin$ $K\left(\nu_{0}\right)$. This last condition implies that there is an $a^{\prime} \in A$ such that $u_{0}\left(a_{0}, \nu_{0}\right)<$ $u_{0}\left(a^{\prime}, \nu_{0}\right)$. Denote $\varepsilon=u_{0}\left(a^{\prime}, \nu_{0}\right)-u_{0}\left(a_{0}, \nu_{0}\right)$.

Since $u_{n} \rightarrow u_{0}$ uniformly with $n \rightarrow \infty$ there exists a number $N_{1} \in \mathbb{N}$ such that for all $n>N_{1}$ and all $(a, \nu) \in A \times M(A)$

$$
\left|u_{0}(a, \nu)-u_{n}(a, \nu)\right|<\frac{\varepsilon}{4} .
$$

On the other hand the continuity of $u_{0}$ implies that there exists $N_{2} \in \mathbb{N}$ such that for each $n>N_{2}$ it hold

$$
\left|u_{0}\left(a_{0}, \nu_{0}\right)-u_{0}\left(a_{n}, \nu_{n}\right)\right|<\frac{\varepsilon}{4} \quad \text { and } \quad\left|u_{0}\left(a^{\prime}, \nu_{0}\right)-u_{0}\left(a^{\prime}, \nu_{n}\right)\right|<\frac{\varepsilon}{4} .
$$

Thus, if $n>\max \left\{N_{1}, N_{2}\right\}$ we obtain

$$
\begin{aligned}
u_{n}\left(a_{n}, \nu_{n}\right) & <u_{0}\left(a_{n}, \nu_{n}\right)+\frac{\varepsilon}{4}<u_{0}\left(a_{0}, \nu_{0}\right)+\frac{\varepsilon}{2} \\
& =u_{0}\left(a^{\prime}, \nu_{0}\right)-\frac{\varepsilon}{2}<u_{0}\left(a^{\prime}, \nu_{n}\right)-\frac{\varepsilon}{4}<u_{n}\left(a^{\prime}, \nu_{n}\right) .
\end{aligned}
$$

This condition however contradicts the assumption that $\left(u_{n}, a_{n}\right) \in K\left(\nu_{n}\right)$. Hence, $\left(u_{0}, a_{0}\right) \in K\left(\nu_{0}\right)$ and this implies the upper-semicontinuity of the correspondence $K$.

Lemma 4.2. The correspondence $B: M(A) \rightarrow M(\mathcal{U} \times A)$ is upper-semicontinuous.

Proof. Let $\nu_{n} \in M(A)$ for all $n \in \mathbb{N}, \nu \in M(A)$ and $\nu_{n} \rightarrow \nu$. Consider arbitrary sequence $\tau_{n}^{\prime} \in M(\mathcal{U} \times A)$ which converges to $\tau^{\prime} \in M(\mathcal{U} \times A)$ with $n \rightarrow \infty$ and covers $\left\{\nu_{n}\right\}_{n \in \mathbb{N}}$, i.e. $\tau_{n}^{\prime} \in B\left(\nu_{n}\right)$ for every $n \in \mathbb{N}$. Let us assume that $\tau^{\prime} \notin B(\nu)$, that is $\tau^{\prime}(K(\nu))<1$. This means that there exists $\left(u_{0}, a_{0}\right) \in \operatorname{supp}\left(\tau^{\prime}\right) \subset \mathcal{U} \times A$ such that

$$
\left(u_{0}, a_{0}\right) \notin K(\nu) .
$$

On the other hand, since $\tau_{n}^{\prime} \in B\left(\nu_{n}\right)$ we have that

$$
\operatorname{supp}\left(\tau_{n}^{\prime}\right) \subset K\left(\nu_{n}\right)
$$

for every $n \in \mathbb{N}$. Lower semicontinuity of the map supp (see Lemma 2.9) implies that there exists a sequence $\left(u_{n}, a_{n}\right)_{n \in \mathbb{N}}$ converging to $\left(u_{0}, a_{0}\right)$ such that $\left(u_{n}, a_{n}\right) \in \operatorname{supp}\left(\tau_{n}^{\prime}\right)$ for every $n \in \mathbb{N}$ and, according to (4.2) $\left(u_{n}, a_{n}\right) \in K\left(\nu_{n}\right)$. Upper-semicontinuity of the 
correspondence $K$ (see Lemma 4.1) implies however that $\lim _{n \rightarrow \infty}\left(u_{n}, a_{n}\right)=\left(u_{0}, a_{0}\right) \in K(\nu)$ which contradicts with (4.1). Thus, $\tau^{\prime} \in B(\nu)$ and therefore the correspondence $B$ is upper-semicontinuous.

Mas-Colell (1986) proved that the set CNE of all CN equilibria of an additive anonymous game is not empty. We now consider the analogous result for CN equilibria in non-additive anonymous games. The proof is similar in spirit to that given by MasColell (1986) and is based on the topological results stated above.

Theorem 4.3. Let $\mu$ be a non-additive anonymous game. Then $\mathrm{CNE} \neq \emptyset$.

Proof. Since $\mathcal{U}$ and $A$ are compact Hausdorff spaces, so are $M(\mathcal{U})$ and $M(\mathcal{U} \times A)$. The set $B_{1}^{\prime}(\mu)$ is compact and convex subset of $M(\mathcal{U} \times A)$ and the set $K\left(\tau_{A}\right)$ is closed in $\mathcal{U} \times A$. Define a correspondence $\Phi: B_{1}^{\prime}(\mu) \rightarrow B_{1}^{\prime}(\mu)$ by $\Phi(\tau)=B_{1}^{\prime}(\mu) \cap B\left(\tau_{A}\right)$ for each $\tau \in B_{1}^{\prime}(\mu)$. It is clear that $\Phi(\tau)$ is convex and compact for all $\tau$. This correspondence is upper-semicontinuous due to Lemmata 2.6 and 4.2. Therefore, the conditions of the Fan fixed point theorem are satisfied (see Fan (1952)). Hence, there exists $\tau \in \Phi(\tau)$, that is, $\tau\left(K\left(\tau_{A}\right)\right)=1$.

Remark 4.4. Note that in spite of the fact that in the case of additive measure $\mu$, additive equilibria is a subset of non-additive equilibria, the above existence theorem for non-additive games is not a consequence of Mas-Colell's Theorem 1 in general. MasColell's case can only cover situations where beliefs about the player's distribution is additive and does not tell us anything about the existence of equilibrium under uncertainty. Let us look at this in more detail.

First of all let us note that the characteristics spaces $\mathcal{U}$ are not identical for additive and non-additive games. Since $P(A) \subset M(A)$, the set of players characteristics under uncertainty is broader. Each player has to determine the expected payoff in the case if beliefs about opponents actions are non-additive and this brings more variety to the set of possible payoff functions. Let us denote by $\mathcal{U}^{\prime}$ the set of payoff functions on $A \times P(A)$ defined as restrictions of those from $\mathcal{U}$. In this way we define a continuous restriction map $r: \mathcal{U} \rightarrow \mathcal{U}^{\prime}$.

In order to make a connection between the two types of anonymous games (additive and non-additive) we define a restriction $\mu^{\prime}$ of the capacity $\mu \in M(\mathcal{U})$ on $M\left(\mathcal{U}^{\prime}\right)$ by $\mu^{\prime}=M r(\mu)$. If $\mu$ happens to be additive (there is no uncertainty in the game), so is its restriction $\mu^{\prime}$. Thus, if $\tau^{\prime} \in P\left(\mathcal{U}^{\prime} \times A\right)$ is an equilibrium for the game $\mu^{\prime}$, one can easily construct an equilibrium $\tau \in P(\mathcal{U} \times A)$ for $\mu$ by $\tau(E)=\tau^{\prime}\left(E \cap\left(\mathcal{U}^{\prime} \times A\right)\right)$. Thus, in 
the case of an additive capacity we do not need to prove a separate existence theorem. However, if $\mu$ is not additive, its restriction $\mu^{\prime}$ might not be additive in general and hence Mas-Colell's theorem would not be applicable. This motivates the use of nonadditive equilibria in the presence of uncertainty and shows that Mas-Colell's concept of equilibrium does not always work.

\section{Symmetric equilibria}

An important property of CN equilibria, considered by Mas-Colell (1986), is their symmetry.

Definition 5.1. A $C N$ equilibrium $\tau$ for a game $\mu$ is symmetric if there is a measurable function $f: \mathcal{U} \rightarrow A$ such that $\tau(\operatorname{graph}(f))=1$.

Under a symmetric equilibrium distribution players with the same characteristic take the same action from $A$. In fact, a symmetric equilibrium in an anonymous game is analogous to a pure strategy equilibrium in a finite player game. The function $f$ assigns to each characteristic $u$ from $\mathcal{U}$ a unique action $a$ from $A$ that all players with characteristic $u$ choose under this equilibrium. Thus, existence of a symmetric equilibrium in the anonymous additive game implies existence of a pure strategy equilibrium.

There is a difference between symmetric equilibrium in additive and non-additive anonymous games. In additive games payers will play strategies that are not specified by function $f$ with probability 0 while a symmetric equilibrium does not rule out possibilities of other strategies in non-additive games due to presence of uncertainty.

Coming back to the example in Section 3.1, equilibrium distribution $\tau^{*}$ is symmetric. There exists function $f:\{1,2\} \rightarrow\{\mathbb{R}, L\}$, defined by $f(1)=R, f(2)=L$, which satisfies the condition of Definition 5.1. Indeed, $\operatorname{graph}(f)=\{(1, R),(2, L)\}$ and it is shown that $\tau^{*}(\operatorname{graph}(f))=1$. In this equilibrium each player has optimistic beliefs according to which he will be matched with an opponent of type 1 . In this case a player of type 1 is better off if he plays $R$ and a player of type two receives higher payoff under strategy $L$.

Although, a CN equilibrium distribution always exists for additive anonymous games, there may be no symmetric equilibrium in general. Mas-Colell (1986) showed that if the game $\mu$ is atomless and the action set is finite, then there is a symmetric Cournot-Nash equilibrium. 
The concept of non-additive anonymous games allow us to establish a much more general result. The sufficient condition when a $\mathrm{CN}$ equilibrium distribution can be symmetrized is given as follows.

Definition 5.2. A $C N$ equilibrium $\tau$ for a game $\mu$ can be symmetrized if there exists a symmetric $C N$ equilibrium distribution $\tau^{\prime}$ such that $K\left(\tau_{A}\right)=K\left(\tau_{A}^{\prime}\right)$.

Let $\tau \in M(\mathcal{U} \times A)$ be a $\mathrm{CN}$ equilibrium distribution for a non-additive game $\mu$. Consider the correspondence $B_{\tau}: \mathcal{U} \rightarrow A$ acting by the formula $B_{\tau}(u)=\{a \in$ $\left.A:(u, a) \in K\left(\tau_{A}\right)\right\}$.

In the case of additive anonymous games, Khan and Sun (1987) prove that every CN equilibrium distribution can be symmetrized if $\mu$ is atomless and finite. Later, Khan and Sun (1995) relaxed the finiteness assumption and extended the result up to countable compact action spaces. Rath, Sun and Yamashige (1995) go further and establish a more general result which states that each $\mathrm{CN}$ equilibrium distribution $\tau$ of an atomless game can be symmetrized if the set $D=\left\{\mu \circ f^{-1}: f\right.$ is a measurable selection from $\left.B_{\tau}\right\}$ is closed. However, in all these cases, the game is required to be atomless. In the nonadditive case, this condition is not necessary any more.

Proposition 5.3. If there is a Borel selection $f: \mathcal{U} \rightarrow A$ of $B_{\tau}$ such that

$$
\tau_{A}(\operatorname{im}(f))=1
$$

then $\tau$ can be symmetrized.

Proof. Let us construct a symmetric CN equlibrium capacity $\tau^{\prime}$ satisfying the condition of Definition 5.2. For any Borel set $G \in \mathcal{U} \times A$ we set $\tau^{\prime}(G)=\tau(G)$ if $\operatorname{graph}(f) \nsubseteq G$ and $\tau^{\prime}(G)=1$ if $\operatorname{graph}(f) \subseteq G$.

Let us note first that $\tau^{\prime}$ is a capacity. Indeed, for any $F, G \in \mathcal{U} \times A$ such that $F \subset G$ we have $\tau^{\prime}(F)=\tau(F) \leq \tau(G)=\tau^{\prime}(G)$ if $\operatorname{graph}(f) \nsubseteq G, \tau^{\prime}(F)=\tau(F) \leq 1=\tau^{\prime}(G)$ if $f(\mathcal{U}) \nsubseteq F$ and $\operatorname{graph}(f) \subseteq F, \tau^{\prime}(F)=1=\tau^{\prime}(G)$ if $\operatorname{graph}(f) \subseteq F$.

Let us show that $K\left(\tau_{A}^{\prime}\right)=K\left(\tau_{A}\right)$. As the set $K\left(\tau_{A}\right)$ depends on the marginal $\tau_{A}$, we have to show that this marginal does not change while switching from capacity $\tau$ to $\tau^{\prime}$. Let $F \subset A$ such that $\operatorname{im}(f) \subset F$. According to the condition of the proposition, $\tau(F)=1=\tau^{\prime}(F)$. If $\operatorname{im}(f) \nsubseteq F$ then $\operatorname{graph}(f) \nsubseteq \mathcal{U} \times F$ which implies that $\tau(\mathcal{U} \times F)=$ $\tau^{\prime}(\mathcal{U} \times F)$ by the construction of $\tau^{\prime}$. Thus, $\tau_{A}^{\prime}(F)=\tau_{A}(F)$.

Finally, $\tau^{\prime}$ is a CN equilibrium capacity, since $\operatorname{im}(f) \subseteq K\left(\tau_{A}\right)=K\left(\tau_{A}^{\prime}\right)$ and hence $\tau^{\prime}\left(K\left(\tau_{A}^{\prime}\right)\right)=1$. 
Remark 5.4. By definition, the capacity of the set of actions $\operatorname{im}(f)$, which players use in the new symmetrized equilibrium, has to be equal to 1 . The condition $\tau_{A}(\operatorname{im}(f))=1$ makes sure that it is possible to modify the equilibrium $\tau$ without changing its marginal distribution $\tau_{A}$.

By definition of the equilibrium, $\tau\left(K\left(\tau_{A}\right)\right)=1$. As a result, according to the construction of the correspondence $B_{\tau}$ one finds that $\tau_{A}\left(\operatorname{im}\left(B_{\tau}\right)\right)=1$. This means that all actions which are in the set of pay-off maximizing actions have a total (nonadditive) measure of 1 .

When the equilibrium distribution (capacity) $\tau$ is being symmetrized, the same condition should hold for the symmetrized equilibrium $\tau^{\prime}$. By the concept of symmetrization, we do not want to change the marginal $\tau_{A}$ and therefore the condition $\tau_{A}^{\prime}=\tau_{A}$ should be satisfied. Thus, one should be able to find a selection from the correspondence $B_{\tau}$ such that all actions from the set of pay-off maximizing actions should have in total measure 1.

For example, let $A=\left\{a_{1}, a_{2}\right\}$ and $\mathcal{U}=\left\{u_{1}, u_{2}\right\}$ and let $\tau$ being the equilibrium such that $K\left(\tau_{A}\right)=\left\{\left(a_{1}, u_{1}\right),\left(a_{2}, u_{1}\right),\left(a_{2}, u_{2}\right)\right\}$ and $\tau_{A}\left(a_{2}\right)<1$. There exist two possible selections: either $f\left(u_{1}\right)=a_{1}$ and $f\left(u_{2}\right)=a_{2}$ or $f\left(u_{1}\right)=a_{2}$ and $f\left(u_{2}\right)=a_{2}$. If one decides to use the second option to construct the symmetrized equilibrium $\tau^{\prime}$, this will destroy the condition $\tau_{A}^{\prime}=\tau_{A}$ since $\tau^{\prime}\left(a_{2}\right)=1$.

Remark 5.5. An additive game with atoms may not have a symmetric CNED. The following example is due to Rath et al. (1995). Consider a game with the action set $A=\left\{a_{1}, a_{2}\right\}$ and let $u \in \mathcal{U}$ be given by $u\left(a_{1}, \nu\right)=\frac{1}{2}, u\left(a_{2}, \nu\right)=1-\nu\left(a_{2}\right)$ with $\nu \in P(A)$. Let $\mu=\delta_{u}$ denote the game (a Dirac measure concentrated on $u$ ).

Let $\tau$ be a CNED of $\mu$. Suppose $\tau_{A}\left(a_{2}\right)<\frac{1}{2}$. Then $u\left(a_{1}, \tau_{A}\right)<u\left(a_{2}, \tau_{A}\right) \Rightarrow$ $\tau\left(K\left(\tau_{A}\right)\right)=\tau\left(u, a_{2}\right)=1 \Rightarrow \tau_{A}\left(a_{2}\right)=1$ and we get a contradiction. Hence, $\tau_{A}\left(a_{2}\right) \nless \frac{1}{2}$. In the same way we can conclude that $\tau_{A}\left(a_{2}\right) \ngtr \frac{1}{2}$ and thus, the only possible case is $\tau_{A}\left(a_{1}\right)=\frac{1}{2}$ and $\tau_{A}\left(a_{2}\right)=\frac{1}{2}$. The only equilibrium distribution $\tau$ is that defined by $\tau\left(\left\{u, a_{1}\right\}\right)=\frac{1}{2}$ and $\tau\left(\left\{u, a_{2}\right\}\right)=\frac{1}{2}$. Since every player has the same utility function, the space of characteristics is a singleton $\{u\}$ and $B_{\tau}(u)=\left\{a_{1}, a_{2}\right\}$. There may be only two possible selections: $f(u)=a_{1}$ or $f(u)=a_{2}$ each of them has graph of measure $\frac{1}{2}$. This implies $\tau$ is not symmetric and cannot be symmetrized.

If one allows distributions to be non-additive, then a symmetric equilibrium can be easily constructed. For example, capacity $\tau\left(u, a_{1}\right)=1, \tau\left(u, a_{2}\right)=\frac{3}{4}$ is a CN equilibrium capacity for $\mu$, as the selection $f$ which maps $u$ to $a_{1}$ has a graph $\left\{\left(u, a_{1}\right)\right\}$ of capacity 
1. However, it is worth noting that not every $\mathrm{CN}$ equilibrium can be symmetrized. For instance, a capacity defined by $\tau\left(u, a_{1}\right)=\tau\left(u, a_{2}\right)=\frac{1}{2}$ (which is additive) cannot be symmetrized even under non-additive beliefs (the same argument as above applies).

Therefore, the previous result holds under weaker conditions than in the additive case. This can be seen from the proof of the Theorem from Khan and Sun (1987). They in fact show how to construct the selection which satisfies the condition of Proposition 5.3 for games with atomless space of players' characteristics and finite number of actions. Under uncertainty, a CN equilibrium can be symmetrized for games with any compact space of actions (not necessarily finite) and it also does not require the space of characteristics to be atomless.

\section{Upper-semicontinuity of CN Equilibria}

Having proved the existence theorem, we now consider a sequence of non-additive games to study how variations in players' beliefs affects the set of equilibria. The second result of the paper is given in the following theorem, where we investigate the sensitivity of the CN equilibrium to the initial parameter. It asserts then when the beliefs vary "continuously", the set of CN equilibria varies upper-semicontinuously.

Theorem 6.1. The $C N$ equilibria correspondence $\mathrm{CNE}: M(\mathcal{U}) \rightarrow M(\mathcal{U} \times A)$ is uppersemicontinuous.

Proof. Let us define a correspondence $B_{1}: M(\mathcal{U}) \times M(A) \rightarrow M(\mathcal{U} \times A)$ by $B_{1}\left(\mu, \tau_{A}\right)=$ $B_{1}^{\prime}(\mu) \cap B_{1}^{\prime \prime}\left(\tau_{A}\right)$. The fact that the correspondence $B_{1}$ is continuous implies from the open-multicommutativity of the functor $M$, which is proved in Kozhan (2006).

The set of all CN equilibria of the game $\mu$ can be represented as CNE $=\underset{\tau_{A} \in M(A)}{\cup}\left(B_{1}\left(\mu, \tau_{A}\right) \cap\right.$ $B\left(\tau_{A}\right)$ ). Lemmata 2.6, 2.7 and 4.2 imply that the correspondence CNE is uppersemicontinuous.

The statement of Theorem 6.1 says that if we consider a sequence of games $\left\{\mu_{k}\right\}_{k \in \mathbb{N}}$ with $\mu_{k} \in M(\mathcal{U})$ which converges to $\mu^{*}$ and a sequence if their CN equilibria $\left\{\tau_{k}\right\}_{k \in \mathbb{N}}$ such that $\tau_{k} \in \operatorname{CNE}\left(\mu_{k}\right) \subset M(\mathcal{U} \times A)$ which converges to $\tau^{*}$ then $\tau^{*}$ is a $\mathrm{CN}$ equilibria for the game $\mu^{*}$ (that is, $\tau^{*} \in \operatorname{CNE}\left(\mu^{*}\right)$ ). This means that small deviations from the initial beliefs will not decrease the set of CN equilibria. 


\section{Conclusion}

Much of economic theory studies situations which can be modelled by anonymous games. Many models of market games involve the notion of perfect competition, where no participant has the market power to influence prices. The framework of anonymous games developed by Schmeidler (1973) provides the required tools to model this situation.

This paper considers an extension of anonymous games to the case where players are faced with Knightian Uncertainty. In particular, it is assumed that there are two types of uncertainty: about possible strategies of opponents and about the distribution over agents' characteristics involved in the game. Both types of uncertainty have been modelled using capacities, which are generalizations of probability distributions. This paper has generalized Mas-Colell's (Mas-Colell 1986) equilibrium concept for anonymous games to the case of games under uncertainty. We have established the existence theorem of CN equilibria for such games. A sufficient condition is given under which a CN equilibrium can be symmetrized. This condition is weaker than for additive anonymous games.

Moreover, we have shown that the $\mathrm{CN}$ equilibrium correspondence which maps initial beliefs about the distribution over agents' characteristics into the set of $\mathrm{CN}$ equilibria is upper-semicontinuous. This fact could be useful when considering approximations and investigating stability and sensitivity of the set of CN equilibria.

The notion of uncertainty brings more flexibility into the structure of CN equilibria of the games. A clear advantage is that we can incorporate more psychological aspects into the model which will reflect the real world more realistically. Players are allowed to construct their beliefs in optimistic or pessimistic manners, to be uncertainty averse or uncertainty loving. The cost is the increase in the number of equilibria in the game, although some equilibria can be easily filtered out as unrealistic.

Another issue which concerns capacities is how to use them in real games. The problem is that up to our knowledge there is no randomization device which could generate realizations from a non-additive distribution. Since there is uncertainty in the model, there is also uncertainty in the equilibrium and in what a realization of the equilibrium will be. A non-additive equilibrium distribution gives us a general picture of how equilibrium strategies would look on average. A non-additive equilibrium enables us to see how the players will tend to play. This has a strong relation to the notion of fuzzy sets - one could see where approximately the set is, but cannot 
precisely determine its boundaries. How to play a capacity strategy remains an open and challenging question.

\section{Acknowledgements}

I express my gratitude to Dionysius Glycopantis, Allan Muir, Mark Salmon and Kostas Koufopoulos for the numerous remarks and valuable suggestions.

\section{References}

Aliprantis, Ch., Glycopantis, D. and Puzzello, D.: 2006, The joint continuity of the expected payoffs functions, Journal of Mathematical Economics, bf 42, 121-130.

Camerer, C. and Ho, T.: 1994, Violations of the betweenness of axiom and nonlinearity in probability, Journal of Risk and Uncertainty 8, 167-196.

Choquet, G.: 1953, Theory of capacities, Annales de l'Institut Fourier 5, 131-295.

Curley, S. and Yates, F.: 1989, An empirical evaluation of descriptive models of ambuguity reactions in choice situations, Journal of Mathematical Psychology 33, 397427.

Denneberg, D.: 1994, Non-additive Measure and Integral, Kluwer Academic Publishers, , Dordrecht.

Dow, J. and Werlang, S.: 1994, Nash equilibrium under Knightian Uncertainty: Breaking down backward induction, Journal of Economic Theory 64(2), 305-324.

Eichberger, J., Kelsey, D.: 2000, Non-Additive Beliefs and Strategic Equilibria, Games and Economic Behaviour 30, 183-215.

Eilenberg, S. and Moore, J.: 1965, Adjoint functors and triples, Illinois Journal of Mathematics 9, 381-398.

Ellsberg, D.: 1961, Risk, ambiguity and the Savage axioms, Quarterly Journal of Economics 75, 643-669.

Fan, K.: 1952, Fixed-point minimax theorems in locally convex topological linear spaces, Proceedings of the National Academy of Sciences 38, 121-126. 
Ghirardato, P., LeBreton, M.: 2000, Choquet Rationality, Journal of Economic Theory 90, 277-285.

Gilboa, I. and Schmeidler, D.: 1989, Maxmin expected utility with non-unique prior, Journal of Mathematical Ecoomics 18, 141-153.

Glycopantis, D., and Muir, A.: 1999, Nash equilibria in $\infty$-dimensional spaces: an approximation theorem, Economic Theory 13, 743-751.

Glycopantis, D., and Muir, A.: 2000, Continuiuty of the payoff function, Economic Theory, 16, 239-244.

Glycopantis, D. and Muir, A.: 2008, Nash equilibria with Knightian uncertainty; the case of capacities, Economic Theory, forthcoming.

Green, E.: 1984, Continuum and finite-player noncooperative models of competition, Econometrica 52(4), 975-994.

Groes, E., Jacobsen, H., Sloth, B., Tranaes, T.: 1998, Nash Equilibrium in Lower Probabilities, Theory and Decision 44, 37-66.

Hart, S., Hildenbrand, W. and Kohlberg, E.: 1974, On equilibrium allocations as distributions on the commodity space, Journal of Mathematical Economics, 1, 159-166.

Hildenbrand, W.: 1974, Core and Equilibria of a Large Economy, Princeton University Press, Princeton, New Jarsey.

Hogarth, R. and Einhorn, H.: 1990, Venture theory: A model of desicion weights, Management Science 36, 780-803.

Kajii, A. and Morris, S.: 1998, Payoff continuity in incomplete information games, Journal of Economic Theory 82, 267-276.

Khan, A. and Sun, Y.: 1995, Extremal structures and symmetric equilibria with countable actions, Journal of Mathematical Economics 24, 239-248.

Khan, A. and Sun, Y.: 1987, On symmetric Cournot-Nash equilibrium distributions in a finite-action, atomless game, In: Equilibrium theory in infinite dimensional spaces, Eds.: Khan, A. and Yennelis, N., Sprimger-Verlag, Berlin.

Klibanoff, P.: 1996, Uncertainty, decisions, and normal games, Mimeo. 
Knight, F.: 1921, Risk, Uncertainty and Profit, Houghton Miffin, Boston.

Kozhan, R.: 2006, Open-multicommutativity of the functor of capacities, Visnyk of Lviv University. Series Mechanical and Mathematical, 70. Available from www.arxiv.org, Working Paper Nr.: math.GN/0610678.

Kozhan, R. and Zarichnyi, M.: 2008, Nash equilibria for games in capacities, Economic Theory 35, 321-331.

Lo, K.: 1996, Equilibrium in beliefs under uncertainty, Journal of Economic Theory 71, 443-484.

Lyons, R.: 2001, The Microstructure Approach to Exchange Rates, The MIT Press, Boston.

Marinacci, M.: 2000, Ambiguous Games, Games and Economic Behaviour 31, 191-219.

Mas-Colell, A.: 1986, On a theorem of Schmeidler, Journal of Mathematical Economics 13.

Michael, E.: 1951, Topologies on spaces of subsets, Transactions of American Mathematical Sosiety 71, 152-182.

Milgrom, P. and Weber, R.: 1985, Distributional strategies for games with incomplete information, Mathematics of Operations Research 10(4), 619-632.

Mukerji, S.: 1997, Understanding the Nonadditive Probability Decision Model, Economic Theory 9, 23-46.

Nykyforchyn, O. and Zarichnyi, M.: 2006, The functor of capacities in the catogery of compact hausdorff spaces, Preprint.

Rath, K., Sun, Y. and Yamashige, S.: 1995, The nonexistence of symmetric equilibria in anonymous games with compact action spaces, Journal of Mathematical Economics 24, 331-346

Savage, L.: 1954, The Foundation of Statistics, John Willey and Sons, New York.

Schmeidler, D.: 1973, Equilibrium points of nonatomic games, Journal of Statistical Physics 17(4), 295-300. 
Schmeidler, D.: 1989, Subjective probability and expected utility without additivity, Econometrica 57, 571-587.

Teleiko, A. and Zarichnyi, M.: 1999, Categorical Topology of Compact Hausdorf Spaces, Vol. 5 of Mathematical Studii, Monograph Series, VNTL Publishers.

Tversky, A. and Fox, C.: 1995, Weighting risk and uncertainty, Psychological Review 102, 269-283.

Zarichnyi, M.: 1990, On covariant topological functors, I, Questions and Answers in General Topology 8, 317-369.

Zhou, L.: 1998, Integral representation of continuous comonotonically additive functionals, Transactions of American Mathematical Society 350(5), 1811-1822. 


\section{List of other working papers:}

\section{8}

1. Roman Kozhan and Rozalia Pal, Firms' Investment under Financial Constraints: A Euro Area Investigation, WP08-07

2. Roman Kozhan and Mark Salmon, On Uncertainty, Market Timing and the Predictability of Tick by Tick Exchange Rates, WP08-06

3. Roman Kozhan and Mark Salmon, Uncertainty Aversion in a Heterogeneous Agent Model of Foreign Exchange Rate Formation, WP08-05

4. Roman Kozhan, Non-Additive Anonymous Games, WP08-04

5. Thomas Lux, Stochastic Behavioral Asset Pricing Models and the Stylized Facts, WP08-03

6. Reiner Franke, A Short Note on the Problematic Concept of Excess Demand in Asset Pricing Models with Mean-Variance Optimization, WP08-02

7. Alexandra Dias, Semi-parametric estimation of joint large movements of risky assets, WP08-01

\section{7}

1. Timur Yusupov and Thomas Lux, The Efficient Market Hypothesis through the Eyes of an Artificial Technical Analyst: An Application of a New Chartist Methodology to High-Frequency Stock Market Data, WP07-13

2. Liu Ruipeng, Di Matteo and Thomas Lux, True and Apparent Scaling: The Proximity of the Markov- Switching Multifractal Model to Long-Range Dependence, WP07-12

3. Thomas Lux, Rational Forecasts or Social Opinion Dynamics? Identification of Interaction Effects in a Business Climate Survey, WP07-11

4. Thomas Lux, Collective Opinion Formation in a Business Climate Survey, WP07-10

5. Thomas Lux, Application of Statistical Physics in Finance and Economics, WP07-09

6. Reiner Franke, A Prototype Model of Speculative Dynamics With Position-Based Trading, WP07-08

7. Reiner Franke, Estimation of a Microfounded Herding Model On German Survey Expectations, WP07-07

8. Cees Diks and Pietro Dindo, Informational differences and learning in an asset market with boundedly rational agents, WP07-06

9. Markus Demary, Who Do Currency Transaction Taxes Harm More: Short-Term Speculators or Long-Term Investors?, WP07-05

10. Markus Demary, A Heterogenous Agents Model Usable for the Analysis of Currency Transaction Taxes, WP07-04

11. Mikhail Anufriev and Pietro Dindo, Equilibrium Return and Agents' Survival in a Multiperiod Asset Market: Analytic Support of a Simulation Model, WP07-03

12. Simone Alfarano and Michael Milakovic, Should Network Structure Matter in Agent-Based Finance?, WP07-02

13. Simone Alfarano and Reiner Franke, A Simple Asymmetric Herding Model to Distinguish Between Stock and Foreign Exchange Markets, WP07-01

\section{6}

1. Roman Kozhan, Multiple Priors and No-Transaction Region, WP06-24

2. Martin Ellison, Lucio Sarno and Jouko Vilmunen, Caution and Activism? Monetary Policy Strategies in an Open Economy, WP06-23

3. Matteo Marsili and Giacomo Raffaelli, Risk bubbles and market instability, WP06-22

4. Mark Salmon and Christoph Schleicher, Pricing Multivariate Currency Options with Copulas, WP06-21 
5. Thomas Lux and Taisei Kaizoji, Forecasting Volatility and Volume in the Tokyo Stock Market: Long Memory, Fractality and Regime Switching, WP06-20

6. Thomas Lux, The Markov-Switching Multifractal Model of Asset Returns: GMM Estimation and Linear Forecasting of Volatility, WP06-19

7. Peter Heemeijer, Cars Hommes, Joep Sonnemans and Jan Tuinstra, Price Stability and Volatility in Markets with Positive and Negative Expectations Feedback: An Experimental Investigation, WP06-18

8. Giacomo Raffaelli and Matteo Marsili, Dynamic instability in a phenomenological model of correlated assets, WP06-17

9. Ginestra Bianconi and Matteo Marsili, Effects of degree correlations on the loop structure of scale free networks, WP06-16

10. Pietro Dindo and Jan Tuinstra, A Behavioral Model for Participation Games with Negative Feedback, WP06-15

11. Ceek Diks and Florian Wagener, A weak bifucation theory for discrete time stochastic dynamical systems, WP06-14

12. Markus Demary, Transaction Taxes, Traders' Behavior and Exchange Rate Risks, WP06-13

13. Andrea De Martino and Matteo Marsili, Statistical mechanics of socio-economic systems with heterogeneous agents, WP06-12

14. William Brock, Cars Hommes and Florian Wagener, More hedging instruments may destabilize markets, WP06-11

15. Ginwestra Bianconi and Roberto Mulet, On the flexibility of complex systems, WP06-10

16. Ginwestra Bianconi and Matteo Marsili, Effect of degree correlations on the loop structure of scale-free networks, WP06-09

17. Ginwestra Bianconi, Tobias Galla and Matteo Marsili, Effects of Tobin Taxes in Minority Game Markets, WP06-08

18. Ginwestra Bianconi, Andrea De Martino, Felipe Ferreira and Matteo Marsili, Multi-asset minority games, WP06-07

19. Ba Chu, John Knight and Stephen Satchell, Optimal Investment and Asymmetric Risk for a Large Portfolio: A Large Deviations Approach, WP06-06

20. Ba Chu and Soosung Hwang, The Asymptotic Properties of AR(1) Process with the Occasionally Changing AR Coefficient, WP06-05

21. Ba Chu and Soosung Hwang, An Asymptotics of Stationary and Nonstationary AR(1) Processes with Multiple Structural Breaks in Mean, WP06-04

22. Ba Chu, Optimal Long Term Investment in a Jump Diffusion Setting: A Large Deviation Approach, WP06-03

23. Mikhail Anufriev and Gulio Bottazzi, Price and Wealth Dynamics in a Speculative Market with Generic Procedurally Rational Traders, WP06-02

24. Simonae Alfarano, Thomas Lux and Florian Wagner, Empirical Validation of Stochastic Models of Interacting Agents: A "Maximally Skewed" Noise Trader Model?, WP06-01

\section{5}

1. Shaun Bond and Soosung Hwang, Smoothing, Nonsynchronous Appraisal and CrossSectional Aggreagation in Real Estate Price Indices, WP05-17

2. Mark Salmon, Gordon Gemmill and Soosung Hwang, Performance Measurement with Loss Aversion, WP05-16

3. Philippe Curty and Matteo Marsili, Phase coexistence in a forecasting game, WP05-15

4. Matthew Hurd, Mark Salmon and Christoph Schleicher, Using Copulas to Construct Bivariate Foreign Exchange Distributions with an Application to the Sterling Exchange Rate Index (Revised), WP05-14

5. Lucio Sarno, Daniel Thornton and Giorgio Valente, The Empirical Failure of the Expectations Hypothesis of the Term Structure of Bond Yields, WP05-13

6. Lucio Sarno, Ashoka Mody and Mark Taylor, A Cross-Country Financial Accelorator: Evidence from North America and Europe, WP05-12

7. Lucio Sarno, Towards a Solution to the Puzzles in Exchange Rate Economics: Where Do We Stand?, WP05-11

8. James Hodder and Jens Carsten Jackwerth, Incentive Contracts and Hedge Fund Management, WP05-10

9. James Hodder and Jens Carsten Jackwerth, Employee Stock Options: Much More Valuable Than You Thought, WP05-09 
10. Gordon Gemmill, Soosung Hwang and Mark Salmon, Performance Measurement with Loss Aversion, WP05-08

11. George Constantinides, Jens Carsten Jackwerth and Stylianos Perrakis, Mispricing of S\&P 500 Index Options, WP05-07

12. Elisa Luciano and Wim Schoutens, A Multivariate Jump-Driven Financial Asset Model, WP0506

13. Cees Diks and Florian Wagener, Equivalence and bifurcations of finite order stochastic processes, WP05-05

14. Devraj Basu and Alexander Stremme, CAY Revisited: Can Optimal Scaling Resurrect the (C)CAPM?, WP05-04

15. Ginwestra Bianconi and Matteo Marsili, Emergence of large cliques in random scale-free networks, WP05-03

16. Simone Alfarano, Thomas Lux and Friedrich Wagner, Time-Variation of Higher Moments in a Financial Market with Heterogeneous Agents: An Analytical Approach, WP05-02

17. Abhay Abhayankar, Devraj Basu and Alexander Stremme, Portfolio Efficiency and Discount Factor Bounds with Conditioning Information: A Unified Approach, WP05-01

\section{4}

1. Xiaohong Chen, Yanqin Fan and Andrew Patton, Simple Tests for Models of Dependence Between Multiple Financial Time Series, with Applications to U.S. Equity Returns and Exchange Rates, WP04-19

2. Valentina Corradi and Walter Distaso, Testing for One-Factor Models versus Stochastic Volatility Models, WP04-18

3. Valentina Corradi and Walter Distaso, Estimating and Testing Sochastic Volatility Models using Realized Measures, WP04-17

4. Valentina Corradi and Norman Swanson, Predictive Density Accuracy Tests, WP04-16

5. Roel Oomen, Properties of Bias Corrected Realized Variance Under Alternative Sampling Schemes, WP04-15

6. Roel Oomen, Properties of Realized Variance for a Pure Jump Process: Calendar Time Sampling versus Business Time Sampling, WP04-14

7. Richard Clarida, Lucio Sarno, Mark Taylor and Giorgio Valente, The Role of Asymmetries and Regime Shifts in the Term Structure of Interest Rates, WP04-13

8. Lucio Sarno, Daniel Thornton and Giorgio Valente, Federal Funds Rate Prediction, WP04-12

9. Lucio Sarno and Giorgio Valente, Modeling and Forecasting Stock Returns: Exploiting the Futures Market, Regime Shifts and International Spillovers, WP04-11

10. Lucio Sarno and Giorgio Valente, Empirical Exchange Rate Models and Currency Risk: Some Evidence from Density Forecasts, WP04-10

11. Ilias Tsiakas, Periodic Stochastic Volatility and Fat Tails, WP04-09

12. Ilias Tsiakas, Is Seasonal Heteroscedasticity Real? An International Perspective, WP04-08

13. Damin Challet, Andrea De Martino, Matteo Marsili and Isaac Castillo, Minority games with finite score memory, WP04-07

14. Basel Awartani, Valentina Corradi and Walter Distaso, Testing and Modelling Market Microstructure Effects with an Application to the Dow Jones Industrial Average, WP04-06

15. Andrew Patton and Allan Timmermann, Properties of Optimal Forecasts under Asymmetric Loss and Nonlinearity, WP04-05

16. Andrew Patton, Modelling Asymmetric Exchange Rate Dependence, WP04-04

17. Alessio Sancetta, Decoupling and Convergence to Independence with Applications to Functional Limit Theorems, WP04-03

18. Alessio Sancetta, Copula Based Monte Carlo Integration in Financial Problems, WP04-02

19. Abhay Abhayankar, Lucio Sarno and Giorgio Valente, Exchange Rates and Fundamentals: Evidence on the Economic Value of Predictability, WP04-01

\section{2}

1. Paolo Zaffaroni, Gaussian inference on Certain Long-Range Dependent Volatility Models, WP02-12

2. Paolo Zaffaroni, Aggregation and Memory of Models of Changing Volatility, WP02-11

3. Jerry Coakley, Ana-Maria Fuertes and Andrew Wood, Reinterpreting the Real Exchange Rate - Yield Diffential Nexus, WP02-10 
4. Gordon Gemmill and Dylan Thomas, Noise Training, Costly Arbitrage and Asset Prices: evidence from closed-end funds, WP02-09

5. Gordon Gemmill, Testing Merton's Model for Credit Spreads on Zero-Coupon Bonds, WP0208

6. George Christodoulakis and Steve Satchell, On th Evolution of Global Style Factors in the MSCI Universe of Assets, WP02-07

7. George Christodoulakis, Sharp Style Analysis in the MSCI Sector Portfolios: A Monte Caro Integration Approach, WP02-06

8. George Christodoulakis, Generating Composite Volatility Forecasts with Random Factor Betas, WP02-05

9. Claudia Riveiro and Nick Webber, Valuing Path Dependent Options in the Variance-Gamma Model by Monte Carlo with a Gamma Bridge, WP02-04

10. Christian Pedersen and Soosung Hwang, On Empirical Risk Measurement with Asymmetric Returns Data, WP02-03

11. Roy Batchelor and Ismail Orgakcioglu, Event-related GARCH: the impact of stock dividends in Turkey, WP02-02

12. George Albanis and Roy Batchelor, Combining Heterogeneous Classifiers for Stock Selection, WP02-01

\section{1}

1. Soosung Hwang and Steve Satchell, GARCH Model with Cross-sectional Volatility; GARCHX Models, WP01-16

2. Soosung Hwang and Steve Satchell, Tracking Error: Ex-Ante versus Ex-Post Measures, WP01-15

3. Soosung Hwang and Steve Satchell, The Asset Allocation Decision in a Loss Aversion World, WP01-14

4. Soosung Hwang and Mark Salmon, An Analysis of Performance Measures Using Copulae, WP01-13

5. Soosung Hwang and Mark Salmon, A New Measure of Herding and Empirical Evidence, WP01-12

6. Richard Lewin and Steve Satchell, The Derivation of New Model of Equity Duration, WP0111

7. Massimiliano Marcellino and Mark Salmon, Robust Decision Theory and the Lucas Critique, WP01-10

8. Jerry Coakley, Ana-Maria Fuertes and Maria-Teresa Perez, Numerical Issues in Threshold Autoregressive Modelling of Time Series, WP01-09

9. Jerry Coakley, Ana-Maria Fuertes and Ron Smith, Small Sample Properties of Panel Timeseries Estimators with I(1) Errors, WP01-08

10. Jerry Coakley and Ana-Maria Fuertes, The Felsdtein-Horioka Puzzle is Not as Bad as You Think, WP01-07

11. Jerry Coakley and Ana-Maria Fuertes, Rethinking the Forward Premium Puzzle in a Nonlinear Framework, WP01-06

12. George Christodoulakis, Co-Volatility and Correlation Clustering: A Multivariate Correlated ARCH Framework, WP01-05

13. Frank Critchley, Paul Marriott and Mark Salmon, On Preferred Point Geometry in Statistics, WP01-04

14. Eric Bouyé and Nicolas Gaussel and Mark Salmon, Investigating Dynamic Dependence Using Copulae, WP01-03

15. Eric Bouyé, Multivariate Extremes at Work for Portfolio Risk Measurement, WP01-02

16. Erick Bouyé, Vado Durrleman, Ashkan Nikeghbali, Gael Riboulet and Thierry Roncalli, Copulas: an Open Field for Risk Management, WP01-01

\section{0}

1. Soosung Hwang and Steve Satchell, Valuing Information Using Utility Functions, WP00-06

2. Soosung Hwang, Properties of Cross-sectional Volatility, WP00-05

3. Soosung Hwang and Steve Satchell, Calculating the Miss-specification in Beta from Using a Proxy for the Market Portfolio, WP00-04

4. Laun Middleton and Stephen Satchell, Deriving the APT when the Number of Factors is Unknown, WP00-03 
5. George A. Christodoulakis and Steve Satchell, Evolving Systems of Financial Returns: AutoRegressive Conditional Beta, WP00-02

6. Christian S. Pedersen and Stephen Satchell, Evaluating the Performance of Nearest Neighbour Algorithms when Forecasting US Industry Returns, WP00-01

\section{9}

1. Yin-Wong Cheung, Menzie Chinn and Ian Marsh, How do UK-Based Foreign Exchange Dealers Think Their Market Operates?, WP99-21

2. Soosung Hwang, John Knight and Stephen Satchell, Forecasting Volatility using LINEX Loss Functions, WP99-20

3. Soosung Hwang and Steve Satchell, Improved Testing for the Efficiency of Asset Pricing Theories in Linear Factor Models, WP99-19

4. Soosung Hwang and Stephen Satchell, The Disappearance of Style in the US Equity Market, WP99-18

5. Soosung Hwang and Stephen Satchell, Modelling Emerging Market Risk Premia Using Higher Moments, WP99-17

6. Soosung Hwang and Stephen Satchell, Market Risk and the Concept of Fundamental Volatility: Measuring Volatility Across Asset and Derivative Markets and Testing for the Impact of Derivatives Markets on Financial Markets, WP99-16

7. Soosung Hwang, The Effects of Systematic Sampling and Temporal Aggregation on Discrete Time Long Memory Processes and their Finite Sample Properties, WP99-15

8. Ronald MacDonald and Ian Marsh, Currency Spillovers and Tri-Polarity: a Simultaneous Model of the US Dollar, German Mark and Japanese Yen, WP99-14

9. Robert Hillman, Forecasting Inflation with a Non-linear Output Gap Model, WP99-13

10. Robert Hillman and Mark Salmon, From Market Micro-structure to Macro Fundamentals: is there Predictability in the Dollar-Deutsche Mark Exchange Rate?, WP99-12

11. Renzo Avesani, Giampiero Gallo and Mark Salmon, On the Evolution of Credibility and Flexible Exchange Rate Target Zones, WP99-11

12. Paul Marriott and Mark Salmon, An Introduction to Differential Geometry in Econometrics, WP99-10

13. Mark Dixon, Anthony Ledford and Paul Marriott, Finite Sample Inference for Extreme Value Distributions, WP99-09

14. Ian Marsh and David Power, A Panel-Based Investigation into the Relationship Between Stock Prices and Dividends, WP99-08

15. Ian Marsh, An Analysis of the Performance of European Foreign Exchange Forecasters, WP99-07

16. Frank Critchley, Paul Marriott and Mark Salmon, An Elementary Account of Amari's Expected Geometry, WP99-06

17. Demos Tambakis and Anne-Sophie Van Royen, Bootstrap Predictability of Daily Exchange Rates in ARMA Models, WP99-05

18. Christopher Neely and Paul Weller, Technical Analysis and Central Bank Intervention, WP9904

19. Christopher Neely and Paul Weller, Predictability in International Asset Returns: A Reexamination, WP99-03

20. Christopher Neely and Paul Weller, Intraday Technical Trading in the Foreign Exchange Market, WP99-02

21. Anthony Hall, Soosung Hwang and Stephen Satchell, Using Bayesian Variable Selection Methods to Choose Style Factors in Global Stock Return Models, WP99-01

\section{8}

1. Soosung Hwang and Stephen Satchell, Implied Volatility Forecasting: A Compaison of Different Procedures Including Fractionally Integrated Models with Applications to UK Equity Options, WP98-05

2. Roy Batchelor and David Peel, Rationality Testing under Asymmetric Loss, WP98-04

3. Roy Batchelor, Forecasting T-Bill Yields: Accuracy versus Profitability, WP98-03

4. Adam Kurpiel and Thierry Roncalli, Option Hedging with Stochastic Volatility, WP98-02

5. Adam Kurpiel and Thierry Roncalli, Hopscotch Methods for Two State Financial Models, WP98-01 